\title{
BOUNDS ON THE GENERATING FUNCTIONS OF CERTAIN SMOOTHING OPERATIONS
}

\author{
WILLIAM F. TRENCH
}

1. Introduction. Let $H_{k}$ be the space of polynomials of degree not greater than $2 k+1$, and let $f=p+w$, where $p$ is in $H_{k}$ and $w$ is a sample function from a stationary random process with zero mean and spectral density $\psi(\theta)$. We study the problem of filtering $f$ to obtain estimates of $p$ and its derivatives. Consider the polynomial weighting functions

$$
\begin{aligned}
u_{i k}(x) & =\sum_{j=0}^{2 k+1}\left(j+\frac{1}{2}\right) P_{j}^{(2 i)}(0) P_{j}(x), \\
v_{i k}(x) & =\sum_{j=0}^{2 k+1}\left(j+\frac{1}{2}\right) P_{j}^{(2 i+1)}(0) P_{j}(x), \quad 0 \leqq i \leqq n,
\end{aligned}
$$

where $P_{0}, P_{1}, \cdots$ are the Legendre polynomials. Define

$$
g_{i}(x)=\int_{-1}^{1} u_{i k}(y) f(x+y) d y
$$

and

$$
h_{i}(x)=\int_{-1}^{1} v_{i k}(y) f(x+y) d y .
$$

The mean value of $g_{i}(x)$ is given by

$$
E\left[g_{i}(x)\right]=\int_{-1}^{1} u_{i k}(y) p(x+y) d y=p^{(2 i)}(x),
$$

and its random component is

$$
\mu_{i}(x)=\int_{-1}^{1} u_{i k}(y) w(x+y) d y .
$$

The second equality in (2) follows from the well-known reproducing property of the kernel

$$
K_{k}(x, y)=\sum_{j=0}^{2 k+1}\left(j+\frac{1}{2}\right) P_{j}(x) P_{j}(y)
$$

Presented to the Society, August 30, 1966; received by the editors April 29, 1966. 
in $H_{k}$. (More generally, it is well known that if $F$ is square integrable in $[-1,1]$, then

$$
\int_{-1}^{1} K_{k}(x, y) F(y) d y
$$

is the least squares polynomial fit to $F$ of degree not greater than $2 k+1$.) It can be shown that $r_{i}(x)$ is a sample function from a stationary random process with zero mean and spectral density

$$
\alpha_{i}(\theta)=C_{i k}^{2}(\theta) \psi(\theta),
$$

where

$$
C_{i k}(\theta)=\int_{-1}^{1} u_{i k}(x) \cos x \theta d x
$$

It is also known that if the process $w$ is white noise, (that is, if $\psi=$ constant), then $g_{i}(x)$ is the minimum variance estimate of $p^{(2 i)}(x)$. Similarly,

$$
E\left[h_{i}(x)\right]=p^{(2 i+1)}(x),
$$

and the error

$$
p^{(2 i+1)}(x)-h_{i}(x)
$$

has zero mean and spectral density

$$
\beta_{i}(\theta)=S_{i k}^{2}(\theta) \psi(\theta)
$$

where

$$
S_{i k}(\theta)=\int_{-1}^{1} v_{i k}(x) \sin x \theta d x .
$$

The magnitudes of $C_{i k}$ and $S_{i k}$ are of interest because of (3) and (6). In [1], the author established the following theorem.

Theorem 1. For each $k \geqq 0, C_{0 k}(0)=1$, and $\left|C_{0 k}(\theta)\right|<1$ if $\theta \neq 0$.

In this paper we prove the following theorem.

ThEOREM 2 . Let $k \geqq 0$, and $0 \leqq i \leqq k$. Then

$$
\left|\theta^{-2 i} C_{i k}(\theta)\right|<1 \text {, }
$$

and

$$
\left|\theta^{-(2 i+1)} S_{i k}(\theta)\right|<1
$$


if $\theta \neq 0$, and

$$
\lim _{\theta \rightarrow 0} \theta^{-2 i} C_{i k}(\theta)=\lim _{\theta \rightarrow 0} \theta^{-(2 i+1)} S_{i k}(\theta)=(-1)^{i} .
$$

2. Proof of Theorem 2. The proof uses Theorem 1 and an induction argument. We need certain lemmas.

Lemma 1. Let

$$
\begin{aligned}
& F_{i}(\theta)=\int_{-1}^{1} x^{2 i} \cos x \theta d x \\
& G_{i}(\theta)=\int_{-1}^{1} x^{2 i+1} \sin x \theta d x
\end{aligned}
$$

and

$$
\sigma_{i}=\int_{-1}^{1} x^{2 i} d x=\left(i+\frac{1}{2}\right)^{-1}
$$

Then

$$
F_{i}(\theta)=\sum_{j=0}^{k} \sigma_{i+j} \frac{C_{j k}(\theta)}{(2 j) !}, \quad 0 \leqq i \leqq k,
$$

and

$$
G_{i}(\theta)=\sum_{j=0}^{k} \sigma_{i+j+1} \frac{S_{j k}(\theta)}{(2 j+1) !}, \quad 0 \leqq i \leqq k .
$$

Proof. Let

$$
f_{k}(x, \theta)=\int_{-1}^{1} K_{k}(x, y) \cos y \theta d y .
$$

Since $f_{k}$ is the projection of $\cos x \theta$ on $H_{k}$,

$$
\int_{-1}^{1}\left[\cos y \theta-f_{k}(y, \theta)\right] y^{2 i} d y=0, \quad 0 \leqq i \leqq k .
$$

By expanding $K_{k}$ in powers of $x$, we find that

$$
f_{k}(x, \theta)=\sum_{j=0}^{k} \frac{C_{j k}(\theta) x^{2 j}}{(2 j) !},
$$

which, when substituted into (14), yields (12). Equation (13) can be found similarly, by considering the projection of $\sin x \theta$ on $H_{k}$. 
LemmA 2. If $C_{k k}(\alpha)=0$, then $C_{i k}(\alpha)=C_{i, k-1}(\alpha), 0 \leqq i \leqq k-1$, and if $S_{k k}(\beta)=0$, then $S_{i k}(\beta)=S_{i, k-1}(\beta), 0 \leqq i \leqq k-1$.

PROoF. If the highest degree term in $f_{k}$ vanishes, then $f_{k}=f_{k-1}$, from elementary properties of least squares polynomials. This implies the first part, and the second is obtained similarly.

LEMma 3. Let

$$
\alpha_{i k}=\left((-1)^{i} \prod_{q=0}^{k}\left(i+q+\frac{1}{2}\right)\right) / i !(k-i) !
$$

and

$$
\beta_{i k}=\left((-1)^{i} \prod_{q=0}^{k}\left(i+q+\frac{3}{2}\right)\right) / i !(k-i) !
$$

Then

$$
C_{i k}(\theta)=(2 i) ! \alpha_{i k} \sum_{j=0}^{k} \alpha_{j k} \sigma_{i+j} F_{j}(\theta)
$$

and

$$
S_{i k}(\theta)=(2 i+1) ! \beta_{i k} \sum_{j=0}^{k} \beta_{j k} \sigma_{i+j+1} G_{j}(\theta) .
$$

Proof. The matrices of the systems (12) and (13) have general elements of the form $\left(a_{i}+b_{j}\right)^{-1}$. Using the method of [2, Vol. 2, pp. $98,299]$, it can be shown that their inverses have $i, j$ th elements given by $\alpha_{i k} \alpha_{j k} \sigma_{i+j}$ and $\beta_{i k} \beta_{j k} \sigma_{i+j+1}$, respectively, where $0 \leqq i, j \leqq k$.

LEMMA 4.

$$
\frac{d}{d \theta}\left\{\theta^{-2 i-1} S_{i, k-1}(\theta)\right\}=-\frac{\beta_{i, k-1}(2 i+1) !}{\alpha_{k k}(2 k) !} C_{k k}(\theta) \theta^{-2 i-1},
$$

and

$$
0 \leqq i \leqq k-1
$$

(17) $\frac{d}{d \theta}\left\{\theta^{-2 i} C_{i k}(\theta)\right\}=-\frac{\alpha_{i k}(2 i) !}{\beta_{k k}(2 k+1) !} S_{k k}(\theta) \theta^{-2 i}, \quad 0 \leqq i \leqq k$.

Proof. To establish (16), we start by dividing (15), with $k$ replaced by $k-1$, by $\theta^{2 i+1}$, and differentiating. Note that 


$$
\begin{aligned}
\frac{d}{d \theta}\left\{G_{j}(\theta) \theta^{-2 i-1}\right\} & =G_{j}^{\prime}(\theta) \theta^{-2 i-1}-(2 i+1) G_{j}(\theta) \theta^{-2 i-2} \\
& =\theta^{-2 i-1}\left(\frac{2 i+2 j+3}{2 j+2} F_{j+1}(\theta)-\frac{2 i+1}{j+1} \frac{\sin \theta}{\theta}\right),
\end{aligned}
$$

where the last equality follows from the relations

$$
G_{j}^{\prime}(\theta)=F_{j+1}(\theta),
$$

and

$$
G_{j}(\theta)=(\sin \theta) /(j+1)-\left(\theta F_{j+1}(\theta)\right) /(2 j+2),
$$

which can be deduced from (10) and (11). Using the fact that

$$
F_{0}(\theta)=(2 \sin \theta) / \theta,
$$

it can now be seen that

$$
\begin{aligned}
& \frac{d}{d \theta}\left(\theta^{-2 i-1} S_{i, k-1}(\theta)\right) \\
& \quad=\frac{(2 i+1) ! \beta_{i, k-1}}{\theta^{2 i+1}}\left\{\sum_{j=0}^{k-1} \frac{\beta_{j, k-1} F_{j+1}(\theta)}{j+1}-\frac{F_{0}(\theta)}{\sigma_{i}} \sum_{j=0}^{k-1} \frac{\beta_{j, k-1} \sigma_{i+j+1}}{j+1}\right\} .
\end{aligned}
$$

It is easy to verify that

$$
\beta_{j, k-1} /(j+1)=-\alpha_{j+1, k} \sigma_{j+k+1}
$$

and

$$
\sum_{j=0}^{k} \alpha_{j k} \sigma_{j+k} \sigma_{i+j}=0, \quad 0 \leqq i \leqq k-1 .
$$

Using these and (19), (16) can be established. Equation (17) can be derived similarly, using the relations

$$
\begin{gathered}
F_{j}^{\prime}(\theta)=-G_{j}(\theta), \quad F_{j}(\theta)=\sigma_{j}\left(\cos \theta+\frac{\theta}{2} G_{j}(\theta)\right), \\
\alpha_{j k} \sigma_{j}=\beta_{j k} \sigma_{k+j+1}
\end{gathered}
$$

and

$$
\sum_{j=0}^{k} \alpha_{j k} \sigma_{i+j} \sigma_{j}=0, \quad 1 \leqq i \leqq k .
$$

Lemma 5. The functions $\theta^{-2 i} C_{i k}(\theta), 0 \leqq i \leqq k$, have the same critical points. Also, the functions $\theta^{-2 i-1} S_{i k}(\theta), 0 \leqq i \leqq k$, have the same critical 
points. Furthermore, if $\phi$ is a critical point of the former, then

$$
-C_{i k}(\phi) / \phi=S_{i-1, k-1}(\phi), \quad 1 \leqq i \leqq k,
$$

and if $\eta$ is a critical point of the latter, then

$$
\frac{S_{i k}(\eta)}{\eta}=C_{i k}(\eta), \quad 0 \leqq i \leqq k .
$$

Proof. From Lemma 4, the critical points of the first set of functions are the zeroes of $S_{k k}$, while the critical points of the second set are the zeroes of $C_{k+1, k+1}$. This implies the first two statements. To prove (21), multiply and divide the $j$ th term on the right of (12) by $\theta^{2 i}$ and differentiate, to obtain

$$
F_{i}^{\prime}(\theta)=\sum_{j=1}^{k} \sigma_{i+j} \frac{C_{j k}(\theta)}{(2 j-1) ! \theta}+\sum_{j=0}^{k} \sigma_{i+j} \frac{\theta^{2 j}}{(2 j) !} \frac{d}{d \theta}\left(\theta^{-2 j} C_{j k}(\theta)\right) .
$$

Let $\theta=\phi$, where $S_{k k}(\phi)=0$, so that the second sum vanishes. Making use of (20), we can write

$$
G_{i}(\phi)=-\sum_{j=0}^{k-1} \sigma_{i+j+1} \frac{C_{j+1, k}(\phi)}{(2 j+1) ! \phi}, \quad 0 \leqq i \leqq k .
$$

Since $S_{k k}(\phi)=0$, we can infer (21) by comparing this system with (13) (with $\theta=\phi$ ), noting that the solution of the latter is unique, and using Lemma 2.

To derive (22), let $C_{k+1, k+1}(\eta)=0$. Use (12) with $k$ replaced by $k+1$ and $\theta=\eta$, and Lemma 2, to conclude that

$$
F_{i}(\eta)=\sum_{j=0}^{k} \sigma_{i+j} \frac{C_{j k}(\eta)}{(2 j) !}, \quad 0 \leqq i \leqq k+1 .
$$

The last $k$ of these equations can be written

$$
F_{i+1}(\eta)=\sum_{j=0}^{k} \sigma_{i+j+1} \frac{C_{j k}(\eta)}{(2 j) !}, \quad 0 \leqq i \leqq k .
$$

Now multiply and divide the $j$ th term on the right side of (13) by $\theta^{2 j+1}$, differentiate as in the derivation of (23), set $\theta=\eta$, and use (18). The result is

$$
F_{i+1}(\eta)=\sum_{j=0}^{k} \sigma_{i+j+1} \frac{S_{j k}(\eta)}{(2 j) ! \eta}, \quad 0 \leqq i \leqq k .
$$

Comparing this with (24) yields (22). 
We can now complete the proof of Theorem 2, by induction. Assume that $k \geqq 1$, and that Theorem 2 holds for $k-1$. This is so if $k=1$, from Theorem 1. Let $\phi$ be a nonzero critical point of $\theta^{-2 i} C_{i k}(\theta)$, and divide both sides of (21) by $\phi^{2 i-1}$ to obtain

$$
-\phi^{-2 i} C_{i k}(\phi)=\phi^{-2 i+1} S_{i-1, k-1}(\phi), \quad 1 \leqq i \leqq k .
$$

From the induction assumption, it now follows that

$$
\left|\theta^{-2 i} C_{i k}(\theta)\right|<1, \quad 1 \leqq i \leqq k,
$$

if $\theta=\phi$, a nonzero critical point of $\theta^{-2 i} C_{i k}(\theta)$, and therefore the inequality holds for all $\theta \neq 0$. (The inequality with $i=0$ does not follow from this argument, but it has already been established in Theorem 1.) Now let $\eta$ be a nonzero critical point of $\theta^{-2 i-1} S_{i k}(\theta)$, and divide both sides of (22) by $\eta^{2 i}$ to find that

$$
\eta^{-2 i-1} S_{i k}(\eta)=\eta^{-2 i} C_{i k}(\eta), \quad 0 \leqq i \leqq k .
$$

We have just established that the right side is less than unity in magnitude. Hence (8) holds for $\theta=\eta$, and therefore for any $\theta \neq 0$.

The limits (9) are obtained by expanding (4) and (7) in powers of $\theta$, integrating term by term, and noting that

$$
\begin{aligned}
& \int_{-1}^{1} u_{i k}(x) x^{2 j} d x=(2 i) ! \delta_{i j}, \\
& \int_{-1}^{1} v_{i k}(x) x^{2 j+1} d x=(2 i+1) ! \delta_{i j}, \quad 0 \leqq i, j \leqq k .
\end{aligned}
$$

\section{REFERENCES}

1. W. F. Trench, On the stability of midpoint smoothing with Legendre polynomials, Proc. Amer. Math. Soc. 18 (1966), 191-199.

2. G. Polya and G. Szego, Aufgaben und Lehrsätze aus der Analysis, Springer, Berlin, 1925.

Drexel Institute of TeChNology 